\title{
Numerical Integrators for Highly Oscillatory Hamiltonian Systems: A Review
}

\author{
David Cohen ${ }^{1}$, Tobias Jahnke ${ }^{2}$, Katina Lorenz ${ }^{1}$, and Christian Lubich ${ }^{1}$ \\ 1 Mathematisches Institut, Univiversität Tübingen, 72076 Tübingen. \{Cohen, \\ Lorenz, Lubich\}@na.uni-tuebingen.de \\ 2 Freie Universität Berlin, Institut für Mathematik II, BioComputing Group, \\ Arnimallee 2-6, 14195 Berlin. Tobias.Jahnke@math.fu-berlin.de
}

Summary. Numerical methods for oscillatory, multi-scale Hamiltonian systems are reviewed. The construction principles are described, and the algorithmic and analytical distinction between problems with nearly constant high frequencies and with time- or state-dependent frequencies is emphasized. Trigonometric integrators for the first case and adiabatic integrators for the second case are discussed in more detail.

\section{Introduction}

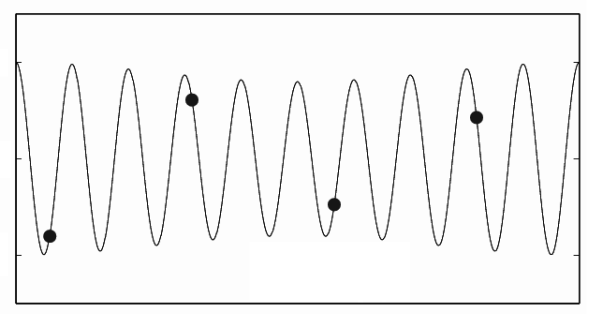

Fig. 1.1. Oscillations and long time steps

Hamiltonian systems with oscillatory solution behaviour are ubiquitous in classical and quantum mechanics. Molecular dynamics, in particular, has motivated many of the new numerical developments in oscillatory Hamiltonian systems in the last decade, though the potential range of their applications goes much farther into oscillatory multi-scale problems of physics and engineering.

Since the publication of the last review article on the numerical solution of oscillatory differential equations by Petzold, Jay \& Yen [PJY97] in 1997, algorithms and their theoretical understanding have developed substantially. This 
fact, together with the pleasure of presenting a final report after six years of funding by the DFG Priority Research Program 1095 on multiscale systems, have incited us to write the present review, which concentrates on Hamiltonian systems. A considerably more detailed (and therefore much longer) account than given here, appears in the second edition of the book by Hairer, Lubich \& Wanner [HLW06, pp. 471-565]. Numerical methods for oscillatory Hamiltonian systems are also treated in the book by Leimkuhler \& Reich [LR04, pp. 257-286], with a different bias from ours.

The outline of this review is as follows. Sect. 2 describes some classes of oscillatory, multi-scale Hamiltonian systems, with the basic distinction between problems with nearly constant and with varying high frequencies. Sect. 3 shows the building blocks with which integrators for oscillatory systems have been constructed. As is illustrated in Fig. 1.1, the aim is to have methods that can take large step sizes, evaluating computationally expensive parts of the system more rarely than a standard numerical integrator which would resolve the oscillations with many small time steps per quasi-period. Sect. 4 deals with trigonometric integrators suited for problems with almost-constant high frequencies, and Sect. 5 with adiabatic integrators for problems with time- or solution-dependent frequencies.

\section{Highly oscillatory Hamiltonian systems}

We describe some problem classes, given in each case by a Hamiltonian function $H$ depending on positions $q$ and momenta $p$ (and possibly on time $t$ ). The canonical equations of motion $\dot{p}=-\nabla_{q} H, \dot{q}=\nabla_{p} H$ are to be integrated numerically.

\subsection{Nearly constant high frequencies}

The simplest example is, of course, the harmonic oscillator given by the Hamiltonian function $H(p, q)=\frac{1}{2} p^{2}+\frac{1}{2} \omega^{2} q^{2}$, with the second-order equation of motion $\ddot{q}=-\omega^{2} q$. This is trivially solved exactly, a fact that can be exploited for constructing methods for problems with Hamiltonian

$$
H(p, q)=\frac{1}{2} p^{T} M^{-1} p+\frac{1}{2} q^{T} A q+U(q)
$$

with a positive semi-definite constant stiffness matrix $A$ of large norm, with a positive definite constant mass matrix $M$ (subsequently taken as the identity matrix for convenience), and with a smooth potential $U$ having moderately bounded derivatives.

The chain of particles illustrated in Fig. 2.1 with equal harmonic stiff springs is an example of a system with a single high frequency $1 / \varepsilon$. With the mid-points and elongations of the stiff springs as position coordinates, we have 


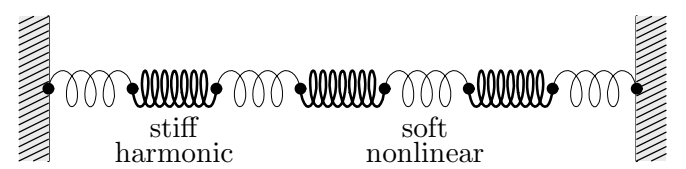

Fig. 2.1. Chain with alternating soft nonlinear and stiff linear springs

$$
A=\frac{1}{\varepsilon^{2}}\left(\begin{array}{cc}
0 & 0 \\
0 & I
\end{array}\right), \quad 0<\varepsilon \ll 1
$$

Other systems have several high frequencies as in

$$
A=\frac{1}{\varepsilon^{2}} \operatorname{diag}\left(0, \omega_{1}, \ldots, \omega_{m}\right), \quad 0<\varepsilon \ll 1,
$$

with $1 \leq \omega_{1} \leq \cdots \leq \omega_{m}$, or a wide range of low to high frequencies without gap as in spatial discretizations of semilinear wave equations.

In order to have near-constant high frequencies, the mass matrix need not necessarily be constant. Various applications lead to Hamiltonians of the form studied by Cohen [Coh06] (with partitions $p=\left(p_{0}, p_{1}\right)$ and $q=\left(q_{0}, q_{1}\right)$ )

$$
H(p, q)=\frac{1}{2} p_{0}^{T} M_{0}(q)^{-1} p_{0}+\frac{1}{2} p_{1}^{T} M_{1}^{-1} p_{1}+\frac{1}{2} p^{T} R(q) p+\frac{1}{2 \varepsilon^{2}} q_{1}^{T} A_{1} q_{1}+U(q)
$$

with a symmetric positive definite matrix $M_{0}(q)$, constant symmetric positive definite matrices $M_{1}$ and $A_{1}$, a symmetric matrix $R(q)$ with $R\left(q_{0}, 0\right)=0$, and a potential $U(q)$. All the functions are assumed to depend smoothly on $q$. Bounded energy then requires $q_{1}=O(\varepsilon)$, so that $p^{T} R(q) p=O(\varepsilon)$, but the derivative of this term with respect to $q_{1}$ is $O(1)$. A simple example of $(2.4)$ is given by a triatomic (water) molecule as illustrated in Fig. 2.2, with strong linear forces that approximately keep the distances and the angle fixed.

\subsection{Explicitly time-dependent high frequencies}

Here the prototype model is the harmonic oscillator with time-dependent frequency, $H(p, q, t)=\frac{1}{2} p^{2}+\frac{1}{2} \varepsilon^{-2} \omega(t)^{2} q^{2}$, with $\omega(t)$ and $\dot{\omega}(t)$ of magnitude $\sim 1$ and $\varepsilon \ll 1$. Solutions of the equation of motion $\ddot{q}=-\varepsilon^{-2} \omega(t)^{2} q$ oscillate with a quasi-period $\sim \varepsilon$, but the frequencies change on the slower time scale $\sim 1$. The action (energy divided by frequency) $I(t)=H(p(t), q(t)) / \omega(t)$ is an almostconserved quantity, called an adiabatic invariant; see, e.g., Henrard [Hen93].

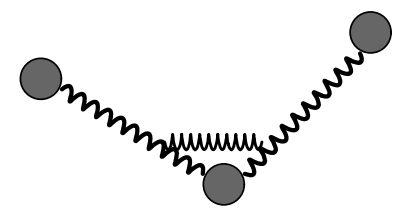

Fig. 2.2. Triatomic molecule 
Numerical methods designed for problems with nearly constant frequencies (and, more importantly, nearly constant eigenspaces) behave poorly on this problem, or on its higher-dimensional extension

$$
H(p, q, t)=\frac{1}{2} p^{T} M(t)^{-1} p+\frac{1}{2 \varepsilon^{2}} q^{T} A(t) q+U(q, t),
$$

which describes oscillations in a mechanical system undergoing a slow driven motion. Here $M(t)$ is a positive definite mass matrix, $A(t)$ is a positive semidefinite stiffness matrix, and $U(q, t)$ is a potential, all of which are assumed to be smooth with derivatives bounded independently of the small parameter $\varepsilon$. This problem again has adiabatic invariants associated with each of its high frequencies as long as the frequencies remain separated. However, on small time intervals where eigenvalues almost cross, rapid non-adiabatic transitions may occur, leading to further numerical challenges.

\subsection{State-dependent high frequencies}

Similar difficulties are present, and related numerical approaches have recently been developed, in problems where the high frequencies depend on the position, as in the problem class studied analytically by Rubin \& Ungar [RU57], Takens [Tak80], and Bornemann [Bor98]:

$$
H(p, q)=\frac{1}{2} p^{T} M(q)^{-1} p+\frac{1}{\varepsilon^{2}} V(q)+U(q),
$$

with a constraining potential $V(q)$ that takes its minimum on a manifold and grows quadratically in non-tangential directions, thus penalizing motions away from the manifold. In appropriate coordinates we have

$$
V(q)=\frac{1}{2} q_{1}^{T} A\left(q_{0}\right) q_{1} \quad \text { for } \quad q=\left(q_{0}, q_{1}\right)
$$

with a positive definite matrix $A\left(q_{0}\right)$.

A multiple spring pendulum with stiff springs as illustrated in Fig. 2.3 is a simple example, with angles as slow variables $q_{0}$ and elongations of stiff springs as fast variables $q_{1}$. In contrast to the triatomic molecule of Fig. 2.2, where also the angle is kept approximately constant, here the frequencies of the high

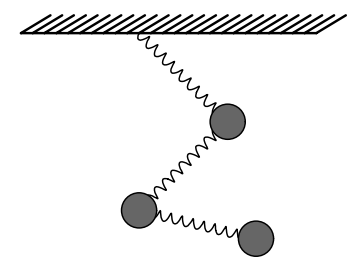

Fig. 2.3. Triple pendulum with stiff springs 
oscillations depend on the angles which change during the motion. Different phenomena occur, and different numerical approaches are appropriate for the two different situations.

As in the case of time-dependent frequencies, difficulties (numerical and analytical) arise when eigenfrequencies cross or come close, which here can lead to an indeterminacy of the slow motion in the limit $\varepsilon \rightarrow 0$ (Takens chaos).

\subsection{Almost-adiabatic quantum dynamics and mixed quantum-classical molecular dynamics}

A variety of new developments in the numerics of oscillatory problems within the last decade were spurred by problems from quantum dynamics; see, e.g., [BN*96, DS03, FL06, HL99b, HL99c, HL03, Jah03, Jah04, JL03, LT05, NR99, NS99, Rei99]. Though these problems can formally be viewed as belonging to the classes treated above, it is worthwhile to state them separately: time-dependent quantum dynamics close to the adiabatic limit is described by an equation

$$
i \varepsilon \dot{\psi}=H(t) \psi
$$

with a finite-dimensional hermitian matrix $H(t)$ with derivatives of magnitude $\sim 1$ representing the quantum Hamiltonian. This is a complex Hamiltonian system with the time-dependent Hamiltonian function $\frac{1}{2} \psi^{*} H(t) \psi$ (consider the real and imaginary parts of $\psi$ as conjugate variables, and take an $\varepsilon^{-1}$ scaled canonical bracket).

A widely used (though disputable) model of mixed quantum-classical mechanics is the Ehrenfest model

$$
\begin{aligned}
\ddot{q} & =-\nabla_{q}\left(\psi^{*} H(q) \psi\right) \\
i \varepsilon \dot{\psi} & =H(q) \psi
\end{aligned}
$$

with a hermitian matrix $H(q)$ depending on the classical positions $q$. This corresponds to the Hamiltonian function $\frac{1}{2} p^{T} p+\frac{1}{2} \psi^{*} H(q) \psi$. The small parameter $\varepsilon$ here corresponds to the square root of the mass ratio of light (quantum) and heavy (classical) particles. While this is indeed small for electrons and nuclei, it is less so for protons and heavy nuclei. In the latter case an adiabatic reduction to just a few eigenstates is not reasonable, and then one has to deal with a quantum Hamiltonian which is a discretization of a Laplacian plus a potential operator that depends on the classical position. Both cases show oscillatory behaviour, but the appropriate numerical treatment is more closely related to that in Sects. 2.3 and 2.1 in the first and second case, respectively. Irrespective of its actual physical modeling qualities, the Ehrenfest model is an excellent model problem for studying numerical approaches and phenomena for nonlinearly coupled slow and fast, oscillatory motion. 


\section{Building-blocks of long-time-step methods: averaging, splitting, linearizing, corotating}

We are interested in numerical methods that can attain good accuracy with step sizes whose product with the highest frequency in the system need not be small; see Fig. 1.1. A large variety of numerical methods to that purpose has been proposed in the last decade, and a smaller variety among them has also been carefully analysed. All these long-time-step and multiscale methods are essentially based on a handful of construction principles, combined in different ways. In addition to those described in the following, time-symmetry of the method has proven to be extremely useful, whereas symplecticity appears to play no essential role in long-time-step methods.

\subsection{Averages}

A basic principle underlying all long-time-step methods for oscillatory differential equations is the requirement to avoid isolated pointwise evaluations of oscillatory functions, but instead to rely on averaged quantities.

Following [HLW06, Sect. VIII.4], we illustrate this for a method for secondorder differential equations such as those appearing in the previous section,

$$
\ddot{q}=f(q), \quad f(q)=f^{[\text {slow }]}(q)+f^{[\text {fast }]}(q) .
$$

The classical Störmer-Verlet method with step size $h$ uses a pointwise evaluation of $f$,

$$
q_{n+1}-2 q_{n}+q_{n-1}=h^{2} f\left(q_{n}\right),
$$

whereas the exact solution satisfies

$$
q(t+h)-2 q(t)+q(t-h)=h^{2} \int_{-1}^{1}(1-|\theta|) f(q(t+\theta h)) d \theta .
$$

The integral on the right-hand side represents a weighted average of the force along the solution, which will now be approximated. At $t=t_{n}$, we replace

$$
f\left(q\left(t_{n}+\theta h\right)\right) \approx f^{[\text {slow }]}\left(q_{n}\right)+f^{[\text {fast }]}(u(\theta h))
$$

where $u(\tau)$ is a solution of the differential equation

$$
\ddot{u}=f^{[\text {slow }]}\left(q_{n}\right)+f^{[\text {fast }]}(u) .
$$

We then have

$$
h^{2} \int_{-1}^{1}(1-|\theta|)\left(f^{[\text {slow }]}\left(q_{n}\right)+f^{[\text {fast }]}(u(\theta h))\right) d \theta=u(h)-2 u(0)+u(-h) .
$$

For the differential equation (3.4) we assume the initial values $u(0)=q_{n}$ and $\dot{u}(0)=\dot{q}_{n}$ or simply $\dot{u}(0)=0$. This initial value problem is solved numerically, e.g., by the Störmer-Verlet method with a micro-step size $\pm h / N$ with 
$N \gg 1$ on the interval $[-h, h]$, yielding numerical approximations $u^{N}( \pm h)$ and $\dot{u}^{N}( \pm h)$ to $u( \pm h)$ and $\dot{u}( \pm h)$, respectively. No further evaluations of $f^{\text {[slow] }}$ are needed for the computation of $u^{N}( \pm h)$ and $\dot{u}^{N}( \pm h)$. This finally gives the symmetric two-step method of Hochbruck \& Lubich [HL99a],

$$
q_{n+1}-2 q_{n}+q_{n-1}=u^{N}(h)-2 u^{N}(0)+u^{N}(-h) .
$$

The method can also be given a one-step formulation, see [HLW06, Sect. VIII.4]. Further symmetric schemes using averaged forces were studied by Hochbruck \& Lubich [HL99c] and Leimkuhler \& Reich [LR01].

The above method is efficient if solving the fast equation (3.4) over the whole interval $[-h, h]$ is computationally less expensive than evaluating the slow force $f^{\text {[slow] }}$. Otherwise, to reduce the number of function evaluations we can replace the average in (3.5) by an average with smaller support,

$$
q_{n+1}-2 q_{n}+q_{n-1}=h^{2} \int_{-\delta}^{\delta} K(\theta)\left(f^{[\text {slow }]}\left(q_{n}\right)+f^{[\text {fast }]}(u(\theta h))\right) d \theta
$$

with $\delta \ll 1$ and an averaging kernel $K(\theta)$ with integral equal to 1 . This is further approximated by a quadrature sum involving the values $f^{[\mathrm{fast}]}\left(u^{N}(m h / N)\right)$ with $|m| \leq M$ and $1 \ll M \ll N$. The resulting method is an example of a heterogeneous multiscale method as proposed by E [E03] and Engquist \& Tsai [ET05], with macro-step $h$ and micro-step $h / N$. Method (3.7) is in between the Störmer-Verlet method (3.2) $(\delta=0)$ and the averaged-force method (3.6) $(\delta=1)$.

In the above methods, the slow force is evaluated, somewhat arbitrarily, at the particular value $q_{n}$ approximating the oscillatory solution $q(t)$. Instead, one might evaluate $f^{\text {[slow] }}$ at an averaged position $\bar{q}_{n}$, defined by solving approximately an approximate equation

$\ddot{u}=f^{[\mathrm{fast}]}(u), \quad u(0)=q_{n}, \dot{u}(0)=0, \quad$ and setting $\quad \bar{q}_{n}=\int_{-\delta}^{\delta} \widetilde{K}(\theta) u(\theta h) d \theta$,

with another averaging kernel $\widetilde{K}(\theta)$ having integral 1. Such an approach was first studied by García-Archilla, Sanz-Serna \& Skeel [GSS99] for the impulse method (see below), and subsequently in [HL99a] for the averaged-force method, in order to reduce the sensitivity to step size resonances in the numerical solution. For that purpose, it turned out that taking $\delta=1$ (or an integer) is essential.

\subsection{Splitting}

The Störmer-Verlet method (see [HLW03]) can be interpreted as approximating the flow $\varphi_{h}^{H}$ of the system with Hamiltonian $H(p, q)=T(p)+V(q)$ with $T(p)=\frac{1}{2} p^{T} p$ by the symmetric splitting 


$$
\varphi_{h / 2}^{V} \circ \varphi_{h}^{T} \circ \varphi_{h / 2}^{V} .
$$

In the situation of a potential $V=V^{[\text {fast }]}+V^{[\text {slow }]}$, we may instead use a different splitting of $H=\left(T+V^{[\text {fast }]}\right)+V^{[\text {slow] }}$ and approximate the flow $\varphi_{h}^{H}$ of the system by

$$
\varphi_{h / 2}^{V^{[\text {[low }}} \circ \varphi_{h}^{T+V^{[\text {fast }}} \circ \varphi_{h / 2}^{V^{[\text {llow] }}} .
$$

This is the impulse method that was proposed in the context of molecular dynamics by Grubmüller, Heller, Windemuth \& Schulten [GH*91] and Tuckerman, Berne \& Martyna [TBM92]:

1. kick: set $p_{n}^{+}=p_{n}-\frac{1}{2} h \nabla V^{[\text {slow] }}\left(q_{n}\right)$

2. oscillate: solve $\ddot{q}=-\nabla V^{[\text {fast }}(q)$ with initial values $\left(q_{n}, p_{n}^{+}\right)$ over a time step $h$ to obtain $\left(q_{n+1}, p_{n+1}^{-}\right)$

3. kick: set $p_{n+1}=p_{n+1}^{-}-\frac{1}{2} h \nabla V^{\text {slow] }}\left(q_{n+1}\right)$.

Step 2 must in general be computed approximately by a numerical integrator with a smaller time step. If the inner integrator is symplectic and symmetric, as it would be for the Störmer-Verlet method, then also the overall method is symplectic and symmetric.

García-Archilla, Sanz-Serna \& Skeel [GSS99] mollify the impulse method by replacing the slow potential $V^{\text {[slow] }}(q)$ by a modified potential $V^{\text {[slow] }}(\bar{q})$, where $\bar{q}$ represents a local average as considered above.

\subsection{Variation of constants formula}

A particular situation arises when the fast forces are linear, as in

$$
\ddot{q}=-A x+g(q)
$$

with a symmetric positive semi-definite matrix $A$ of large norm. With $\Omega=$ $A^{1 / 2}$, the exact solution satisfies

$$
\begin{array}{r}
\left(\begin{array}{c}
q(t) \\
\dot{q}(t)
\end{array}\right)=\left(\begin{array}{cc}
\cos t \Omega & \Omega^{-1} \sin t \Omega \\
-\Omega \sin t \Omega & \cos t \Omega
\end{array}\right)\left(\begin{array}{c}
q_{0} \\
\dot{q}_{0}
\end{array}\right) \\
+\int_{0}^{t}\left(\begin{array}{c}
\Omega^{-1} \sin (t-s) \Omega \\
\cos (t-s) \Omega
\end{array}\right) g(q(s)) d s .
\end{array}
$$

Discretizing the integral in different ways gives rise to various numerical schemes proposed in the literature for treating (3.9) (the earliest references are Hersch [Her58] and Gautschi [Gau61]). This also gives reinterpretations of the methods discussed above when they are applied to (3.9). We consider a class of trigonometric integrators that reduces to the Störmer-Verlet method for $A=0$ and gives the exact solution for $g=0$ [HLW06, Chap. XIII]: 


$$
\begin{aligned}
& q_{n+1}=\cos h \Omega q_{n}+\Omega^{-1} \sin h \Omega \dot{q}_{n}+\frac{1}{2} h^{2} \Psi g\left(\Phi q_{n}\right) \\
& \dot{q}_{n+1}=-\Omega \sin h \Omega q_{n}+\cos h \Omega \dot{q}_{n}+\frac{1}{2} h\left(\Psi_{0} g\left(\Phi q_{n}\right)+\Psi_{1} g\left(\Phi q_{n+1}\right)\right) .
\end{aligned}
$$

Here $\Psi=\psi(h \Omega)$ and $\Phi=\phi(h \Omega)$, where the filter functions $\psi$ and $\phi$ are smooth, bounded, real-valued functions with $\psi(0)=\phi(0)=1$. Moreover, we have $\Psi_{0}=\psi_{0}(h \Omega), \Psi_{1}=\psi_{1}(h \Omega)$ with even functions $\psi_{0}, \psi_{1}$ satisfying $\psi_{0}(0)=\psi_{1}(0)=1$. The method is symmetric if and only if

$$
\psi(\xi)=\operatorname{sinc}(\xi) \psi_{1}(\xi), \quad \psi_{0}(\xi)=\cos (\xi) \psi_{1}(\xi),
$$

where $\operatorname{sinc}(\xi)=\sin (\xi) / \xi$. In addition, the method is symplectic (for $g=$ $-\nabla U)$ if and only if

$$
\psi(\xi)=\operatorname{sinc}(\xi) \phi(\xi) .
$$

The two-step form of the method reads

$$
q_{n+1}-2 \cos (h \Omega) q_{n}+q_{n-1}=h^{2} \Psi g\left(\Phi q_{n}\right) .
$$

Various methods of Sects. 3.1 and 3.2 can be written in this way, with different filters $\Psi$ and $\Phi$, when they are applied to (3.9):

$$
\begin{aligned}
& \psi(\xi)=\operatorname{sinc}^{2}\left(\frac{1}{2} \xi\right) \phi(\xi)=1 \quad \text { Gautschi [Gau61] and averaged method (3.6) } \\
& \psi(\xi)=\operatorname{sinc}(\xi) \quad \phi(\xi)=1 \quad \text { Deuflhard [Deu79] and impulse method (3.8) } \\
& \psi(\xi)=\operatorname{sinc}^{2}(\xi) \quad \phi(\xi)=\operatorname{sinc}(\xi) \text { García-Archilla \& al. [GSS99]: mollified i.m. } \\
& \psi(\xi)=\operatorname{sinc}^{2}(\xi) \quad \phi(\xi)=1 \quad \text { Hairer \& Lubich [HL00] } \\
& \psi(\xi)=\operatorname{sinc}^{3}(\xi) \quad \phi(\xi)=\operatorname{sinc}(\xi) \quad \text { Grimm \& Hochbruck [GH06] }
\end{aligned}
$$

As will be seen in Sect. 4, the choice of the filter functions has a substantial influence on the long-time properties of the method.

\subsection{Transformation to corotating variables}

For problems where the high frequencies and the corresponding eigenspaces depend on time or on the solution, as in (2.5)-(2.8), it is useful to transform to corotating variables in the numerical treatment.

We illustrate the basic procedure for Schrödinger-type equations (2.7) with a time-dependent real symmetric matrix $H(t)$ changing on a time scale $\sim 1$, for which the solutions are oscillatory with almost-period $\sim \varepsilon$. A time-dependent linear transformation $\eta(t)=T_{\varepsilon}(t) \psi(t)$ takes the system to the form

$$
\dot{\eta}(t)=S_{\varepsilon}(t) \eta(t) \quad \text { with } \quad S_{\varepsilon}=\dot{T}_{\varepsilon} T_{\varepsilon}^{-1}-\frac{i}{\varepsilon} T_{\varepsilon} H T_{\varepsilon}^{-1} .
$$

A first approach is to freeze $H(t) \approx H_{*}$ over a time step and to choose the transformation 

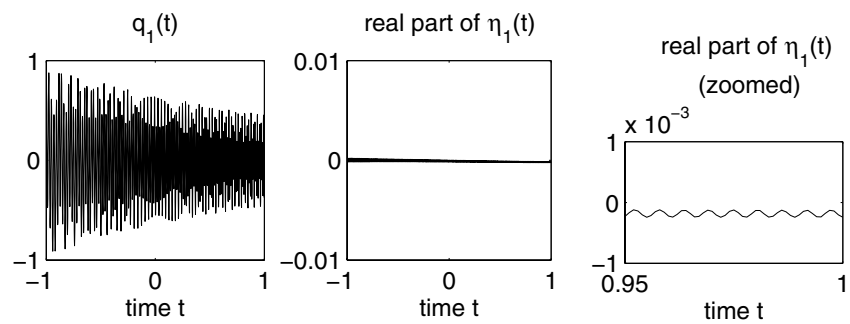

Fig. 3.1. Oscillatory solution component and adiabatic variable as functions of time

$$
T_{\varepsilon}(t)=\exp \left(\frac{i t}{\varepsilon} H_{*}\right)
$$

yielding a matrix function $S_{\varepsilon}(t)$ that is highly oscillatory and bounded in norm by $O(h / \varepsilon)$ for $\left|t-t_{0}\right| \leq h$, if $H_{*}=H\left(t_{0}+h / 2\right)$. Numerical integrators using this transformation together with an appropriate treatment of the oscillatory integrals, are studied by Hochbruck \& Lubich [HL99c], Iserles [Ise02, Ise04], and Degani \& Schiff [DS03]. Step sizes are still restricted by $h=O(\varepsilon)$ in general, but can be chosen larger in the special case when the derivatives of $\frac{1}{\varepsilon} H(t)$ are moderately bounded.

A uniformly bounded matrix $S_{\varepsilon}(t)$ in (3.16) is obtained if we diagonalize

$$
H(t)=Q(t) \Lambda(t) Q(t)^{T}
$$

with a real diagonal matrix $\Lambda(t)=\operatorname{diag}\left(\lambda_{j}(t)\right)$ and an orthogonal matrix $Q(t)$ of eigenvectors depending smoothly on $t$ (possibly except where eigenvalues cross). We define $\eta(t)$ by the unitary adiabatic transformation

$$
\eta(t)=\exp \left(\frac{i}{\varepsilon} \Phi(t)\right) Q(t)^{T} \psi(t) \quad \text { with } \quad \Phi(t)=\operatorname{diag}\left(\phi_{j}(t)\right)=\int_{0}^{t} \Lambda(s) d s,
$$

which represents the solution in a rotating frame of eigenvectors. Such transformations have been in use in quantum mechanics since the work of Born \& Fock [BF28] on the adiabatic invariants $I_{j}(t)=\left|\eta_{j}(t)\right|^{2}$ in Schrödinger equations. Figure 3.1 illustrates the effect of this transformation, showing solution components in the original and in the adiabatic variables.

The transformation (3.17) to adiabatic variables yields a differential equation where the $\varepsilon$-independent skew-symmetric matrix

$$
W(t)=\dot{Q}(t)^{T} Q(t)
$$

is framed by oscillatory diagonal matrices:

$$
\dot{\eta}(t)=\exp \left(\frac{i}{\varepsilon} \Phi(t)\right) W(t) \exp \left(-\frac{i}{\varepsilon} \Phi(t)\right) \eta(t) .
$$

Numerical integrators for (2.7) based on the transformation to the differential equation (3.18) are given by Jahnke \& Lubich [JL03] and Jahnke [Jah04]. The 
simplest of these methods freezes the slow variables $\eta(t)$ and $W(t)$ at the midpoint of the time step, makes a piecewise linear approximation to the phase $\Phi(t)$, and then integrates the resulting system exactly over the time step. This gives the following adiabatic integrator:

$$
\begin{aligned}
& \eta_{n+1}=\eta_{n}+h B\left(t_{n+1 / 2}\right) \frac{1}{2}\left(\eta_{n}+\eta_{n+1}\right) \quad \text { with } \\
& B(t)=\left(\exp \left(-\frac{i}{\varepsilon}\left(\phi_{j}(t)-\phi_{k}(t)\right)\right) \operatorname{sinc}\left(\frac{h}{2 \varepsilon}\left(\lambda_{j}(t)-\lambda_{k}(t)\right)\right) w_{j k}(t)\right)_{j, k} .
\end{aligned}
$$

More involved - and substantially more accurate - methods use a Neumann or Magnus expansion in (3.18) and a quadratic phase approximation. Numerical challenges arise near avoided crossings of eigenvalues, where $\eta(t)$ remains no longer nearly constant and a careful choice of step size selection strategy is needed in order to follow the non-adiabatic transitions; see [JL03] and [HLW06, Chap. XIV].

The extension of this approach to (2.5), (2.6), and (2.8) is discussed in Sect. 5. The transformation to adiabatic variables is also a useful theoretical tool for analysing the error behaviour of multiple time-stepping methods applied to these problems in the original coordinates, such as the impulse and mollified impulse methods considered in Sect. 3.2; see [HLW06, Chap. XIV].

\section{Trigonometric integrators for problems with nearly constant frequencies}

A good understanding of the behaviour of numerical long-time-step methods over several time scales has been gained for Hamiltonian systems with almostconstant high frequencies as considered in Sect. 2.1. We here review results for single-frequency systems (2.1) with (2.2) (and $M=I$ ) from Hairer \& Lubich [HL00] and [HLW06, Chap. XIII], with the particle chain of Fig. 2.1 serving as a concrete example. The variables are split as $q=\left(q_{0}, q_{1}\right)$ according to the blocks in (2.2). We consider initial conditions for which the total energy $H(p, q)$ is bounded independently of $\varepsilon$,

$$
H(p(0), q(0)) \leq \text { Const. }
$$

The principal theoretical tool is a modulated Fourier expansion of both the exact and the numerical solution,

$$
q(t)=\sum_{k} z^{k}(t) e^{i k t / \varepsilon},
$$

an asymptotic multiscale expansion with coefficient functions $z^{k}(t)$ changing on the slow time scale 1 , which multiply exponentials that oscillate with frequency $1 / \varepsilon$. The system determining the coefficient functions turns out to 
have a Hamilton-type structure with formal invariants close to the total and oscillatory energies.

The results on the behaviour of trigonometric integrators (3.15) on different time scales have been extended from single- to multi-frequency systems (possibly with resonant frequencies) by Cohen, Hairer \& Lubich [CHL05], and to systems (2.4) with non-constant mass matrix by Cohen [Coh04, Coh06].

\subsection{Time scale $\varepsilon$}

On this time scale the system (2.1) with (2.2) only shows near-harmonic oscillations with frequency $1 / \varepsilon$ and amplitude $O(\varepsilon)$ in the fast variables $q_{1}$, which are well reproduced by just any numerical integrator.

\subsection{Time scale $\varepsilon^{0}$}

This is the time scale of motion of the slow variables $q_{0}$ under the influence of the potential $U(q)$. Here it is of interest to have an error in the numerical methods which is small in the step size $h$ and uniform in the product of the step size with the high frequency $1 / \varepsilon$. The availability of such uniform error bounds depends on the behaviour of the filter functions $\psi$ and $\phi$ in (3.15) at integral multiples of $\pi$. Under the conditions

$$
\psi(2 k \pi)=\psi^{\prime}(2 k \pi)=0, \quad \psi((2 k-1) \pi)=0, \quad \phi(2 k \pi)=0
$$

for $k=1,2,3, \ldots$, it is shown in [HLW06, Chap. XIII.4] that the error after $n$ time steps is bounded by

$$
\left\|q_{n}-q(n h)\right\| \leq C h^{2}, \quad\left\|\dot{q}_{n}-\dot{q}(n h)\right\| \leq C h \quad \text { for } \quad n h \leq \text { Const. }
$$

with $C$ independent of $h / \varepsilon$ and of bounds of derivatives of the highly oscillatory solution.

Error bounds without restriction of the product of the step size with the frequencies are given for general positive semi-definite matrices $A$ in (2.1) by García-Archilla, Sanz-Serna \& Skeel [GSS99] for the mollified impulse method $\left(\psi(\xi)=\operatorname{sinc}^{2}(\xi), \phi(\xi)=\operatorname{sinc}(\xi)\right)$, by Hochbruck \& Lubich [HL99a] and Grimm [Gri05a] for Gautschi-type methods $\left(\psi(\xi)=\operatorname{sinc}^{2}(\xi / 2)\right.$ and suitable $\phi)$, and most recently by Grimm \& Hochbruck [GH06] for general $A$ and general classes of filter functions $\psi$ and $\phi$.

\subsection{Time scale $\varepsilon^{-1}$}

An energy exchange between the stiff springs in the particle chain takes place on the slower time scale $\varepsilon^{-1}$. To describe this in mathematical terms, let $q_{1, j}$ be the $j$ th component of the fast position variables $q_{1}$, and consider

$$
I_{j}=\frac{1}{2} \dot{q}_{1, j}^{2}+\frac{1}{2 \varepsilon^{2}} q_{1, j}^{2},
$$


which in the example represents the harmonic energy in the $j$ th stiff spring. The quantities $I_{j}$ change on the time scale $\varepsilon^{-1}$. To leading order in $\varepsilon$, their change is described by a differential equation that determines the coefficient of $e^{i t / \varepsilon}$ in the modulated Fourier expansion (4.1). It turns out that for a trigonometric method (3.15), the differential equation for the corresponding coefficient in the modulated Fourier expansion of the numerical solution is consistent with that of the exact solution for all step sizes if and only if

$$
\psi(\xi) \phi(\xi)=\operatorname{sinc}(\xi) \quad \text { for all } \xi \geq 0 .
$$

It is interesting to note that this condition for correct numerical energy exchange is in contradiction with the condition (3.14) of symplecticity of the method, with the only exception of the impulse method, given by $\psi=$ sinc, $\phi=1$. That method, however, does not satisfy (4.2) and is in fact extremely sensitive to near-resonances between frequency and step size $(h / \varepsilon$ near even multiples of $\pi$ ). A way out of these difficulties is to consider trigonometric methods with more than one force evaluation per time step, as is shown in [HLW06, Chap. XIII].

In Fig. 4.1 we show the energy exchange of three numerical methods for the particle chain of Fig. 2.1, with $\varepsilon=0.02$ and with potential and initial data as in [HLW06, p. 22]. At $t=0$ only the first stiff spring is elongated, the other two being at rest position. The harmonic energies $I_{1}, I_{2}, I_{3}$, their sum $I=I_{1}+I_{2}+I_{3}$, and the total energy $H$ (actually $H-0.8$ for graphical reasons) are plotted along the numerical solutions of the following methods, with step sizes $h=0.015$ and $h=0.03$ :

(A) impulse method $(\psi=$ sinc, $\phi=1)$

(B) mollified impulse method $\left(\psi=\operatorname{sinc}^{2}, \phi=\operatorname{sinc}\right)$

(C) heterogeneous multiscale method (3.7) with $\delta=\sqrt{\varepsilon}$.
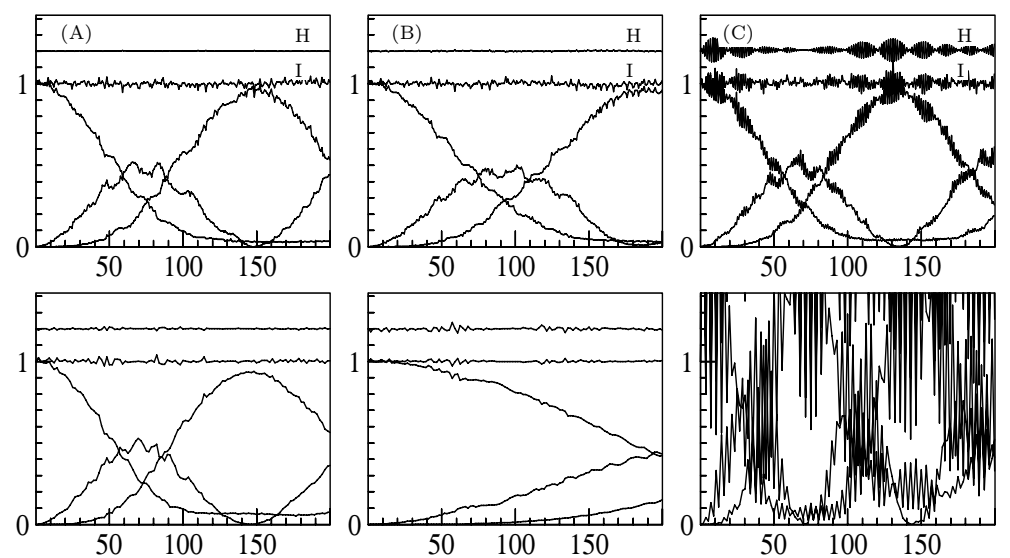

Fig. 4.1. Energy exchange between the stiff springs for methods $(A)-(C)$, with $h=0.015$ (upper) and $h=0.03$ (lower), for $\varepsilon=0.02$ 
For this problem with linear fast forces, the averaging integrals of Sect. 3.1 and the solution of (3.8) are computed exactly. We notice that for the larger step size only method (A) reproduces the energy exchange in a correct way. Method (C) behaves very similarly to the Störmer-Verlet method.

\subsection{Time scales $\varepsilon^{-N}$ with $N \geq 2$}

In Fig. 4.1 it is seen that the total oscillatory energy $I$ remains approximately conserved over long times. Along the exact solution of the problem, $I$ is in fact conserved up to $O(\varepsilon)$ over exponentially long times $t \leq e^{c / \varepsilon}$; see Benettin, Galgani \& Giorgilli [BGG87] and Cohen, Hairer \& Lubich [CHL03] for different proofs based on canonical transformations of Hamiltonian perturbation theory and modulated Fourier expansions (4.1), respectively. Along the numerical solution by a trigonometric integrator (3.15), near-conservation of the oscillatory energy $I$ and the total energy $H$ are shown in [HL00] and [HLW06, Chap. XIII] over times $t \leq \varepsilon^{-N}$ under a non-resonance condition between the frequency and the step-size:

$$
\left|\sin \left(\frac{k h}{2 \varepsilon}\right)\right| \geq c \sqrt{h} \quad \text { for } \quad k=1, \ldots, N .
$$

It is known from [HL00] that the condition

$$
\psi(\xi)=\operatorname{sinc}^{2}(\xi) \phi(\xi)
$$

is necessary to have long-time conservation of the total energy uniformly for all values of $h / \varepsilon$, and numerical experiments indicate that this condition on the filter functions may also be sufficient. Otherwise, energy conservation is lost at least when $h / \varepsilon$ is close to an even multiple of $\pi$.

Figure 4.2 shows, for the same data and methods as before, the maximum deviations of $H$ and $I$ on the interval $[0,1000]$ as functions of $h / \varepsilon$.

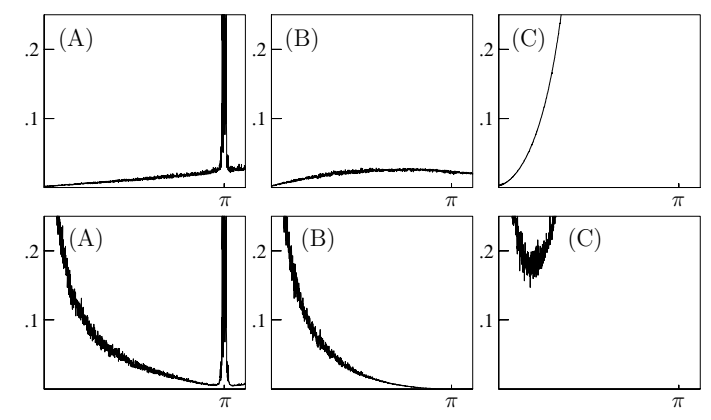

Fig. 4.2. Maximum deviation of total energy (upper) and oscillatory energy (lower) as functions of $h / \varepsilon$ (for step size $h=0.02$ ) 


\section{Adiabatic integrators for problems with varying frequencies}

Adiabatic integrators are a novel class of numerical integrators that have been devised in [JL03, Jah04, Jah03, LJL05, Lor06, HLW06] for various kinds of oscillatory problems with time- or solution-dependent high frequencies, including (2.5)-(2.8). These integrators have in common that the oscillatory part of the problem is transformed to adiabatic variables (cf. Sect. 3.4) and the arising oscillatory integrals are computed analytically or approximated by an appropriate expansion. The methods allow to integrate these oscillatory differential equations with large time steps in the adiabatic regime of wellseparated frequencies and follow non-adiabatic transitions with adaptively refined step sizes.

\subsection{Adiabatic integrators for quantum-classical molecular dynamics}

Following Jahnke [Jah03], we sketch how to construct a symmetric long-timestep method for problem (2.8), which couples in a nonlinear way slow motion and fast oscillations with frequencies depending on the slow variables.

Proceeding as in Sect. 3.4, the quantum system is transformed to adiabatic variables by

$$
\eta(t)=\exp \left(\frac{i}{\varepsilon} \Phi(t)\right) Q(q(t))^{T} \psi(t),
$$

where $H(q)=Q(q) \Lambda(q) Q(q)^{T}$ is a smooth eigendecomposition of the Hamiltonian and

$$
\Phi(t)=\int_{t_{0}}^{t} \Lambda(q(s)) d s, \quad \Phi=\operatorname{diag}\left(\phi_{j}\right)
$$

is the phase matrix, a diagonal matrix containing the time integrals over the eigenvalues $\lambda_{j}(q(t))$ along the classical trajectory. Inserting (5.1) into (2.8) yields the new equations of motion

$$
\begin{aligned}
& \ddot{q}=-\eta^{*} \exp \left(\frac{i}{\varepsilon} \Phi\right) K(q) \exp \left(-\frac{i}{\varepsilon} \Phi\right) \eta \\
& \dot{\eta}=\exp \left(\frac{i}{\varepsilon} \Phi\right) W(q, \dot{q}) \exp \left(-\frac{i}{\varepsilon} \Phi\right) \eta
\end{aligned}
$$

with the tensor $K(q)$ and skew-symmetric matrix $W(q, \dot{q})$ given as

$$
\begin{aligned}
K(q) & =Q(q)^{T} \nabla_{q} H(q) Q(q), \\
W(q, \dot{q}) & =\left(\frac{d}{d t} Q(q)\right)^{T} Q(q)=\left(\nabla_{q} Q(q) \dot{q}\right)^{T} Q(q) .
\end{aligned}
$$


Equations (5.3) and (5.4) can be restated as

$$
\begin{aligned}
& \ddot{q}=-\eta^{*}(E(\Phi) \bullet K(q)) \eta, \\
& \dot{\eta}=(E(\Phi) \bullet W(q, \dot{q})) \eta .
\end{aligned}
$$

where $\bullet$ means entrywise multiplication, and $E(\Phi)$ denotes the matrix

$$
E(\Phi)=\left(e_{j k}(\Phi)\right), \quad e_{j k}(\Phi)=\exp \left(\frac{i}{\varepsilon}\left(\phi_{j}-\phi_{k}\right)\right) .
$$

The classical equation can be integrated using the averaging technique from Sect. 3. We insert (5.6) into (3.3) and, in order to approximate the integral, keep the smooth variables $\eta(t)$ and $K(q(t))$ fixed at the midpoint $t_{n}$ of the interval $\left[t_{n-1}, t_{n+1}\right]$. Since more care is necessary for the oscillating exponentials, we replace $\Phi$ by the linear approximation

$$
\Phi\left(t_{n}+\theta h\right) \approx \Phi\left(t_{n}\right)+\theta h \Lambda\left(q\left(t_{n}\right)\right) .
$$

These modifications yield the averaged Störmer-Verlet method of [Jah03]:

$$
\begin{gathered}
q_{n+1}-2 q_{n}+q_{n-1}=-h^{2} \eta_{n}^{*}\left(E\left(\Phi_{n}\right) \bullet \mathcal{I}\left(q_{n}\right) \bullet K\left(q_{n}\right)\right) \eta_{n} \\
\text { with } \quad \mathcal{I}\left(q_{n}\right)=\int_{-1}^{1}(1-|\theta|) E\left(\theta h \Lambda\left(q_{n}\right)\right) d \theta .
\end{gathered}
$$

The matrix of oscillatory integrals $\mathcal{I}\left(q_{n}\right)$ can be computed analytically: its entries $\mathcal{I}_{j k}\left(q_{n}\right)$ are given by

$$
\begin{aligned}
& \mathcal{I}_{j k}\left(q_{n}\right)=\int_{-1}^{1}(1-|\theta|) \exp \left(i \theta \xi_{j k}\right) d \theta=\operatorname{sinc}^{2}\left(\frac{1}{2} \xi_{j k}\right) \\
& \text { with } \quad \xi_{j k}=\frac{h}{\varepsilon}\left(\lambda_{j}\left(q_{n}\right)-\lambda_{k}\left(q_{n}\right)\right) .
\end{aligned}
$$

Note that in the (computationally uninteresting) small-time-step limit $h / \varepsilon \rightarrow$ 0 the integrator (5.10) converges to the Störmer-Verlet method.

The easiest way to approximate the quantum vector $\eta(t)$ is to keep $\eta(t) \equiv \eta(0)$ simply constant. According to the quantum adiabatic theorem [BF28] the resulting error is only $O(\varepsilon)$ as long as the eigenvalues of $H(q(t))$ are well separated and the eigendecomposition remains smooth. A more reliable method, which in its variable-time-step version follows non-adiabatic transitions in $\eta$ occurring near avoided crossings of eigenvalues, is obtained by integrating Eq. (5.7) from $t_{n}-h$ to $t_{n}+h$, using the linear approximation (5.9) for $\Phi(t)$, and freezing the slow coupling matrix $W(q(t), \dot{q}(t))$ at the midpoint $t_{n}$. This yields the adiabatic integrator from [Jah03], 


$$
\begin{aligned}
& \eta_{n+1}-\eta_{n-1}=2 h\left(E\left(\Phi_{n}\right) \bullet \mathcal{J}\left(q_{n}\right) \bullet W_{n}\right) \eta_{n} \\
& \text { with } \mathcal{J}\left(q_{n}\right)=\frac{1}{2} \int_{-1}^{1} E\left(\theta h \Lambda\left(q_{n}\right)\right) d \theta .
\end{aligned}
$$

The $(j, k)$-entry of the matrix of oscillatory integrals $\mathcal{J}\left(q_{n}\right)$ is simply

$$
\mathcal{J}_{j k}\left(q_{n}\right)=\frac{1}{2} \int_{-1}^{1} \exp \left(i \theta \xi_{j k}\right) d \theta=\operatorname{sinc} \xi_{j k} .
$$

The explicit midpoint rule is recovered in the limit $h / \varepsilon \rightarrow 0$. The derivative contained in (5.5) and the integral in (5.2) are not known explicitly but can be approximated by the corresponding symmetric difference quotient and the trapezoidal rule, respectively. These approximations are denoted by $W_{n}$ and $\Phi_{n}$ in the above formulas.

The approximation properties of method (5.10), (5.12) for large step sizes up to $h \leq \sqrt{\varepsilon}$ are analysed in [Jah03]. A discrete quantum-adiabatic theorem is established, which plays an important role in the error analysis.

\subsection{Adiabatic integrators for problems with time-dependent frequencies}

Adiabatic integrators for mechanical systems with a time-dependent multiscale Hamiltonian (2.5) are presented in [HLW06, Chap. XIV] and [Lor06], following up on previous work by Lorenz, Jahnke \& Lubich [LJL05] for systems (2.5) with $M(t) \equiv I$ and $A(t)$ a symmetric positive definite matrix. To simplify the presentation, we ignore in the following the slow potential and set $U \equiv 0$.

The approach is based on approximately separating the fast and slow time scales by a series of time-dependent canonical linear coordinate transformations, which are done numerically by standard numerical linear algebra routines. The procedure can be sketched as follows:

- The Cholesky decomposition $M(t)=C(t)^{-T} C(t)$ and the transformation $q \mapsto C(t) q$ change the Hamiltonian in such a way that the new mass matrix is the identity.

- The eigendecomposition

$$
A(t)=Q(t)\left(\begin{array}{cc}
0 & 0 \\
0 & \Omega(t)^{2}
\end{array}\right) Q(t)^{T}, \quad \Omega(t)=\operatorname{diag}\left(\omega_{j}(t)\right)
$$

of the symmetric stiffness matrix $A(t)$ allows to split the positions $q=$ $\left(q_{0}, q_{1}\right)$ and momenta $p=\left(p_{0}, p_{1}\right)$ into slow and fast variables $q_{0}, p_{0}$ and $q_{1}, p_{1}$, respectively.

- The fast positions and momenta are rescaled by $\varepsilon^{-1 / 2} \Omega(t)^{1 / 2}$ and by $\varepsilon^{1 / 2} \Omega(t)^{-1 / 2}$, respectively. 
- The previous transforms produce a non-separable term $q^{T} K(t) p$ in the Hamiltonian. One block of the matrix $K(t)$ is of order $O\left(\varepsilon^{-1 / 2}\right)$ and has to be removed by one more canonical transformation.

The Hamiltonian in the new coordinates $p=\left(p_{0}, p_{1}\right)$ and $q=\left(q_{0}, q_{1}\right)$ then takes the form

$$
H(p, q, t)=\frac{1}{2} p_{0}^{T} p_{0}+\frac{1}{2 \varepsilon} p_{1}^{T} \Omega(t) p_{1}+\frac{1}{2 \varepsilon} q_{1}^{T} \Omega(t) q_{1}+q^{T} L(t) p+\frac{1}{2} q^{T} S(t) q
$$

with a lower block-triangular matrix $L$ and a symmetric matrix $S$ of the form

$$
L=\left(\begin{array}{cc}
L_{00} & 0 \\
\varepsilon^{1 / 2} L_{10} L_{11}
\end{array}\right), \quad S=\left(\begin{array}{cc}
S_{00} & \varepsilon^{1 / 2} S_{01} \\
\varepsilon^{1 / 2} S_{10} & \varepsilon S_{11}
\end{array}\right) .
$$

Under the condition of bounded energy, the fast variables $q_{1}$ and $p_{1}$ are now of order $O\left(\varepsilon^{1 / 2}\right)$. The equations of motion read

$$
\begin{aligned}
\dot{p}_{0} & =f_{0}(p, q, t) \\
\dot{q}_{0} & =p_{0}+g_{0}(q, t) \\
\left(\begin{array}{c}
\dot{p}_{1} \\
\dot{q}_{1}
\end{array}\right) & =\frac{1}{\varepsilon}\left(\begin{array}{cc}
0 & -\Omega(t) \\
\Omega(t) & 0
\end{array}\right)\left(\begin{array}{c}
p_{1} \\
q_{1}
\end{array}\right)+\left(\begin{array}{c}
f_{1}(p, q, t) \\
g_{1}(q, t)
\end{array}\right)
\end{aligned}
$$

with functions

$$
\left(\begin{array}{l}
f_{0} \\
f_{1}
\end{array}\right)=-L(t) p-S(t) q, \quad\left(\begin{array}{l}
g_{0} \\
g_{1}
\end{array}\right)=L(t)^{T} q,
$$

which are bounded uniformly in $\varepsilon$. The oscillatory part now takes the form of a skew-symmetric matrix multiplied by $1 / \varepsilon$, similar to $(2.7)$. We diagonalize this matrix and define the diagonal phase matrix $\Phi$ as before:

$$
\begin{array}{cc}
\left(\begin{array}{cc}
0 & -\Omega(t) \\
\Omega(t) & 0
\end{array}\right)=\Gamma i \Lambda(t) \Gamma^{*}, & \Gamma=\frac{1}{\sqrt{2}}\left(\begin{array}{cc}
I & I \\
-i I & i I
\end{array}\right), \\
\Lambda(t)=\left(\begin{array}{cc}
\Omega(t) & 0 \\
0 & -\Omega(t)
\end{array}\right), & \Phi(t)=\int_{t_{0}}^{t} \Lambda(s) d s .
\end{array}
$$

The transformation to adiabatic variables is now taken as

$$
\eta=\varepsilon^{-1 / 2} \exp \left(-\frac{i}{\varepsilon} \Phi(t)\right) \Gamma^{*}\left(\begin{array}{c}
p_{1} \\
q_{1}
\end{array}\right)
$$

with the factor $\varepsilon^{-1 / 2}$ introduced such that $\eta=O(1)$. The equations of motion become

$$
\begin{aligned}
& \dot{p}_{0}=-L_{00} p_{0}-S_{00} q_{0}-\varepsilon S_{01} Q_{1} \eta \\
& \dot{q}_{0}=p_{0}+L_{00}^{T} q_{0}+\varepsilon L_{10}^{T} Q_{1} \eta
\end{aligned}
$$


for the slow variables, and

$$
\dot{\eta}=\exp \left(-\frac{i}{\varepsilon} \Phi\right) W \exp \left(\frac{i}{\varepsilon} \Phi\right) \eta-P_{1}^{*}\left(L_{10} p_{0}+S_{10} q_{0}\right)
$$

for the adiabatic variables, where

$$
W=\Gamma^{*}\left(\begin{array}{cc}
-L_{11} & -\varepsilon S_{11} \\
0 & L_{11}^{T}
\end{array}\right) \Gamma, \quad\left(\begin{array}{c}
P_{1} \\
Q_{1}
\end{array}\right)=\Gamma \exp \left(\frac{i}{\varepsilon} \Phi\right) .
$$

Slow and fast degrees of freedom are only weakly coupled, because in the slow equations (5.16) the fast variable $\eta$ always appears with a factor $\varepsilon$. The oscillatory part has the familiar form of a coupling matrix framed by oscillatory exponentials, cf. (3.18) and (5.4). Under a separation condition for the frequencies $\omega_{j}(t)$, the fact that the diagonal of $W$ is of size $O(\varepsilon)$ implies that the expressions $I_{j}=\left|\eta_{j}\right|^{2}$ are adiabatic invariants. $I_{j}$ is the action (energy divided by frequency)

$$
I_{j}=\frac{1}{\omega_{j}}\left(\frac{1}{2} p_{1, j}^{2}+\frac{\omega_{j}^{2}}{2 \varepsilon^{2}} q_{1, j}^{2}\right) .
$$

An adiabatic integrator for (2.5) is obtained by the following splitting (for details see [HLW06, Chap. XIV] and Lorenz [Lor06]):

1. Propagate the slow variables $\left(p_{0}, q_{0}\right)$ with a half-step of the symplectic Euler method. For the oscillatory function $Q_{1}(t)$, replace the evaluation at $t_{n+1 / 2}=t_{n}+h / 2$ by the average

$$
\mathcal{Q}_{1}^{-} \approx \frac{2}{h} \int_{t_{n}}^{t_{n+1 / 2}} Q_{1}(t) d t
$$

obtained with a linear approximation of the phase $\Phi(t)$ and analytic computation of the integral.

2. Propagate the adiabatic variable $\eta$ with a full step of a method of type (3.19) for (5.17).

3. Propagate the slow variables $\left(p_{0}, q_{0}\right)$ with a half-step of the adjoint symplectic Euler method, with an appropriate average of $Q_{1}(t)$.

The approximation properties of this method are analyzed in [HLW06, Chap. XIV], where it is shown that the error over bounded time intervals, in the original variables of (2.5), is of order $O\left(h^{2}\right)$ in the positions and $O(h)$ in the momenta, uniformly in $\varepsilon$ for $h \leq \sqrt{\varepsilon}$. Numerical comparisons with other methods illustrate remarkable benefits of this approach [Lor06, LJL05].

We present numerical illustrations from Lorenz [Lor06] for the timedependent Hamiltonian (2.5) with $M(t) \equiv I$ and

$$
A(t)=\left(\begin{array}{cc}
t+3 & \delta \\
\delta & 2 t+3
\end{array}\right)^{2}
$$



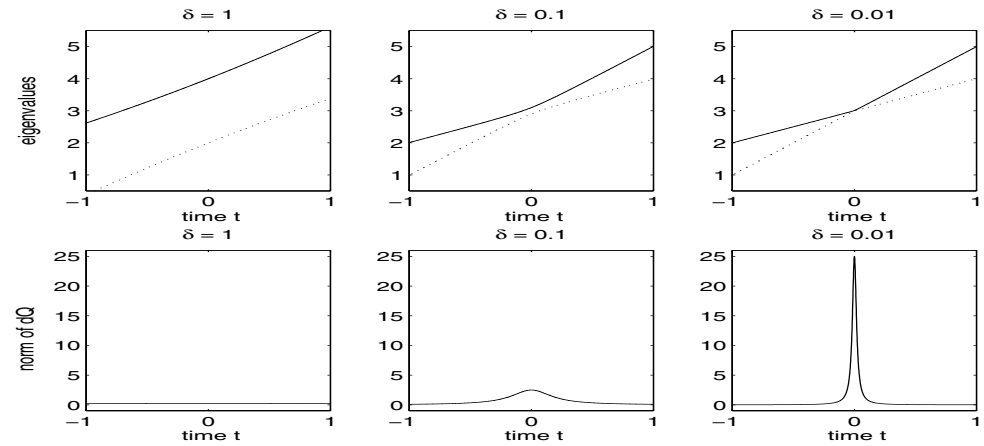

Fig. 5.1. Frequencies $\omega_{j}$ (upper) and $\|\dot{Q}\|$ (lower) for $\delta=1,0.1,0.01$

on the time interval $[-1,1]$. The behaviour of the components of the solution $q(t)$ and the adiabatic variable $\eta(t)$ are as in Fig. 3.1 for $\delta=1$ and $\eta=0.01$.

Figure 5.1 shows the frequencies and the norm of the time derivative of the matrix $Q(t)$ that diagonalizes $A(t)$ for various values of the parameter $\delta$. For small values of $\delta$, the frequencies approach each other to $O(\delta)$ at $t=0$, and $\|\dot{Q}(0)\| \sim \delta^{-1}$. This behaviour affects the adiabatic variables $\eta_{j}(t)$, as is shown in Fig. 5.2.

For $\delta \sim \varepsilon^{1 / 2}$, there appears an $O(1)$ change in $\eta$ in an $O(\delta)$ neighbourhood of $t=0$, and for smaller values of $\delta$ the components of $\eta$ essentially exchange their values; cf. Zener [Zen32] for the analogous situation in Schrödingertype equations (2.7). Small step sizes are needed near $t=0$ to resolve this behaviour. Figure 5.3 shows the step sizes chosen by a symmetric adaptive step selection algorithm described in [HLW06, Chap. XIV] for different values of $\delta$. Errors of similar size are obtained in each case.

\subsection{Integrators for motion under a strong constraining force}

The methods and techniques of the previous subsection can be extended to problems (2.6) with solution-dependent high frequencies [HLW06, Lor06]. The
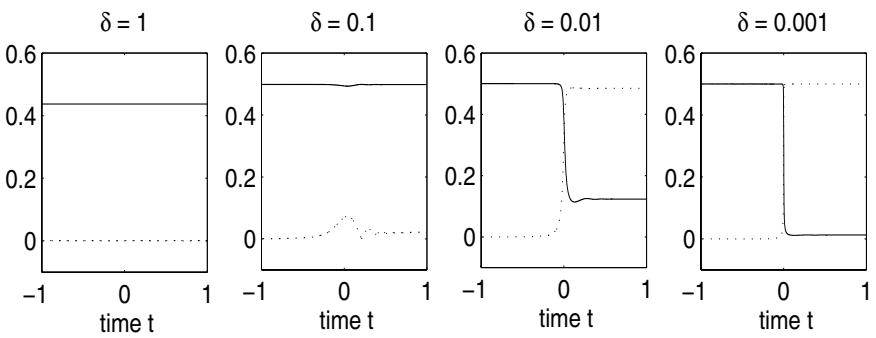

Fig. 5.2. Adiabatic variables $\eta_{j}$ as functions of time 


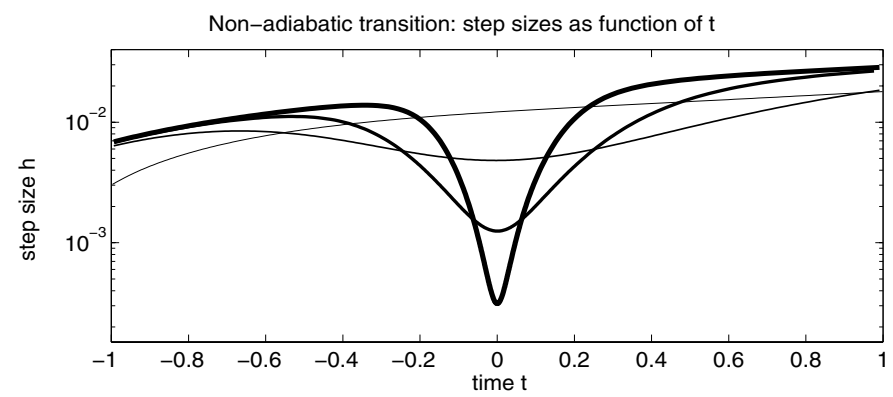

Fig. 5.3. Step size vs. time for $\delta=2^{0}, 2^{-2}, 2^{-4}, 2^{-6}$ (increasingly thicker lines)

procedure is again to apply a series of canonical coordinate transformations to transform (numerically) to a system with nearly-separated slow and fast components:

$$
\begin{aligned}
\dot{p}_{0}= & -\nabla_{q_{0}}\left(\frac{1}{2} p_{0}^{T} M_{0}\left(q_{0}\right)^{-1} p_{0}+U\left(q_{0}, 0\right)\right) \\
& -\nabla_{q_{0}}\left(\frac{1}{2 \varepsilon} p_{1}^{T} \Omega\left(q_{0}\right) p_{1}+\frac{1}{2 \varepsilon} q_{1}^{T} \Omega\left(q_{0}\right) q_{1}\right)+f_{0}(p, q) \\
\dot{q}_{0}= & M_{0}\left(q_{0}\right)^{-1} p_{0}+g_{0}(p, q) \\
\left(\begin{array}{c}
\dot{p}_{1} \\
\dot{q}_{1}
\end{array}\right)= & \frac{1}{\varepsilon}\left(\begin{array}{cc}
0 & -\Omega\left(q_{0}\right) \\
\Omega\left(q_{0}\right) & 0
\end{array}\right)\left(\begin{array}{l}
p_{1} \\
q_{1}
\end{array}\right)+\left(\begin{array}{l}
f_{1}(p, q) \\
g_{1}(p, q)
\end{array}\right)
\end{aligned}
$$

with the diagonal matrix $\Omega\left(q_{0}\right)$ of frequencies $\omega_{j}\left(q_{0}\right)$ and smooth functions of magnitude $f_{0}=O(\varepsilon), g_{0}=O(\varepsilon)$ and $f_{1}=O\left(\varepsilon^{1 / 2}\right), g_{1}=O\left(\varepsilon^{1 / 2}\right)$ in the case of bounded energy and well-separated frequencies. The fast motion of $\left(p_{1}, q_{1}\right)$ is followed numerically in the adiabatic variables $\eta$, which are again defined by (3.17). In these coordinates, the integrator used is then similar to those of Sects. 5.1 and 5.2. Alternatively, the system is integrated in the original coordinates by multiple time-stepping methods such as the (mollified) impulse method of Sect. 3.2, and the above-mentioned transformations are only used as a theoretical tool for analysing the numerical method. We refer to [HLW06, Chap. XIV] for more details.

The actions $I_{j}=\left|\eta_{j}\right|^{2}$ are again adiabatic invariants in the case of wellseparated frequencies, remaining $O\left(\varepsilon^{1 /(m+1)}\right)$ close to their initial values over bounded time intervals if additionally all the expressions $\omega_{j} \pm \omega_{k} \pm \omega_{l}$ have zeros of multiplicity at most $m$. It is worthwhile to note that the oscillatory energy appearing in (5.18) can be written as

$$
\frac{1}{2 \varepsilon} p_{1}^{T} \Omega\left(q_{0}\right) p_{1}+\frac{1}{2 \varepsilon} q_{1}^{T} \Omega\left(q_{0}\right) q_{1}=\sum_{j} I_{j} \omega_{j}\left(q_{0}\right)
$$

so that the limit equation of the slow variables for $\varepsilon \rightarrow 0$ takes the form first discovered by Rubin \& Ungar [RU57], 


$$
\begin{aligned}
& \dot{p}_{0}=-\nabla_{q_{0}}\left(\frac{1}{2} p_{0}^{T} M_{0}\left(q_{0}\right)^{-1} p_{0}+U\left(q_{0}, 0\right)+\sum_{j} I_{j} \omega_{j}\left(q_{0}\right)\right) \\
& \dot{q}_{0}=M_{0}\left(q_{0}\right)^{-1} p_{0}
\end{aligned}
$$

with the oscillatory energy acting as an extra potential. However, as was noted by Takens [Tak80], the slow motion can become indeterminate in the limit $\varepsilon \rightarrow$ 0 when the frequencies do not remain separated; see also Bornemann [Bor98]. In contrast to the integration of the slow limit system with constant actions $I_{j}$, the numerical integration of the full oscillatory system by an adiabatic integrator with adaptive time steps detects changes in the actions. Moreover, it can follow an almost-solution (having small defect in the differential equation) that passes through a non-adiabatic transition.

Acknowlegdments. This work has been supported by the DFG Priority Program 1095 "Analysis, Modeling and Simulation of Multiscale Problems" under LU 532/3-3. In addition to the discussions with participants of this program, we particularly acknowledge those with Assyr Abdulle, Ernst Hairer, and Gerhard Wanner.

\section{References}

[BGG87] G. Benettin, L. Galgani \& A. Giorgilli, Realization of holonomic constraints and freezing of high frequency degrees of freedom in the light of classical perturbation theory. Part I, Comm. Math. Phys. 113 (1987) 87-103.

[BF28] M. Born, V. Fock, Beweis des Adiabatensatzes, Zs. Physik 51 (1928) 165-180.

[Bor98] F. Bornemann, Homogenization in Time of Singularly Perturbed Mechanical Systems, Springer LNM 1687 (1998).

[BN*96] F. A. Bornemann, P. Nettesheim, B. Schmidt, \& C. Schütte, An explicit and symplectic integrator for quantum-classical molecular dynamics, Chem. Phys. Lett., 256 (1996) 581-588.

[BS99] F. A. Bornemann, C. Schütte, On the singular limit of the quantum-classical molecular dynamics model, SIAM J. Appl. Math., 59 (1999) 1208-1224.

[Coh04] D. Cohen, Analysis and numerical treatment of highly oscillatory differential equations, Doctoral Thesis, Univ. de Genève (2004).

[Coh06] D. Cohen, Conservation properties of numerical integrators for highly oscillatory Hamiltonian systems, IMA J. Numer. Anal. 26 (2006) 34-59.

[CHL03] D. Cohen, E. Hairer \& C. Lubich, Modulated Fourier expansions of highly oscillatory differential equations, Found. Comput. Math. 3 (2003) 327-345.

[CHL05] D. Cohen, E. Hairer \& C. Lubich, Numerical energy conservation for multifrequency oscillatory differential equations, BIT 45 (2005) 287-305.

[DS03] I. Degani \& J. Schiff, RCMS: Right correction Magnus series approach for integration of linear ordinary differential equations with highly oscillatory solution, Report, Weizmann Inst. Science, Rehovot, 2003.

[Deu79] P. Deuflhard, A study of extrapolation methods based on multistep schemes without parasitic solutions, Z. angew. Math. Phys. 30 (1979) 177-189.

[E03] W. E, Analysis of the heterogeneous multiscale method for ordinary differential equations, Comm. Math. Sci. 1 (2003) 423-436. 
[ET05] B. Engquist \& Y. Tsai, Heterogeneous multiscale methods for stiff ordinary differential equations, Math. Comp. 74 (2005) 1707-1742.

[FL06] E. Faou \& C. Lubich, A Poisson integrator for Gaussian wavepacket dynamics, Report, 2004. To appear in Comp. Vis. Sci.

[GSS99] B. García-Archilla, J. Sanz-Serna, R. Skeel, Long-time-step methods for oscillatory differential equations, SIAM J. Sci. Comput. 20 (1999) 930-963.

[Gau61] W. Gautschi, Numerical integration of ordinary differential equations based on trigonometric polynomials, Numer. Math. 3 (1961) 381-397.

[Gri05a] V. Grimm, On error bounds for the Gautschi-type exponential integrator applied to oscillatory second-order differential equations, Numer. Math. 100 (2005) 71-89.

[Gri05b] V. Grimm, A note on the Gautschi-type method for oscillatory second-order differential equations, Numer. Math. 102 (2005) 61-66.

[GH06] V. Grimm \& M. Hochbruck, Error analysis of exponential integrators for oscillatory second-order differential equations, J. Phys. A 39 (2006)

[GH*91] H. Grubmüller, H. Heller, A. Windemuth \& K. Schulten, Generalized Verlet algorithm for efficient molecular dynamics simulations with long-range interactions, Mol. Sim. 6 (1991) 121-142.

[HL00] E. Hairer, C. Lubich, Long-time energy conservation of numerical methods for oscillatory differential equations. SIAM J. Num. Anal. 38 (2000) 414-441.

[HLW06] E. Hairer, C. Lubich \& G. Wanner, Geometric Numerical Integration. Structure-Preserving Algorithms for Ordinary Differential Equations. Springer Series in Computational Mathematics 31. 2nd ed., 2006.

[HLW03] E. Hairer, C. Lubich \& G. Wanner, Geometric numerical integration illustrated by the Störmer-Verlet method, Acta Numerica (2003) 399-450.

[Hen93] J. Henrard, The adiabatic invariant in classical mechanics, Dynamics reported, New series. Vol. 2, Springer, Berlin (1993) 117-235.

[Her58] J. Hersch, Contribution à la méthode aux différences, Z. angew. Math. Phys. 9a (1958) 129-180.

[HL99a] M. Hochbruck \& C. Lubich, A Gautschi-type method for oscillatory secondorder differential equations, Numer. Math. 83 (1999) 403-426.

[HL99b] M. Hochbruck \& C. Lubich, A bunch of time integrators for quantum/classical molecular dynamics, in P. Deuflhard et al. (eds.), Computational Molecular Dynamics: Challenges, Methods, Ideas, Springer, Berlin 1999, 421-432.

[HL99c] M. Hochbruck \& C. Lubich, Exponential integrators for quantum-classical molecular dynamics, BIT 39 (1999) 620-645.

[HL03] M. Hochbruck \& C. Lubich, On Magnus integrators for time-dependent Schrödinger equations, SIAM J. Numer. Anal. 41 (2003) 945-963.

[Ise02] A. Iserles, On the global error of discretization methods for highly-oscillatory ordinary differential equations, BIT 42 (2002) 561-599.

[Ise04] A. Iserles, On the method of Neumann series for highly oscillatory equations, BIT 44 (2004) 473-488.

[Jah03] T. Jahnke, Numerische Verfahren für fast adiabatische Quantendynamik, Doctoral Thesis, Univ. Tübingen (2003).

[Jah04] T. Jahnke, Long-time-step integrators for almost-adiabatic quantum dynamics, SIAM J. Sci. Comput. 25 (2004) 2145-2164.

[JL03] T. Jahnke \& C. Lubich, Numerical integrators for quantum dynamics close to the adiabatic limit, Numer. Math. 94 (2003) 289-314. 
[LT05] C. Lasser \& S. Teufel, Propagation through conical crossings: an asymptotic semigroup, Comm. Pure Appl. Math. 58 (2005) 1188-1230.

[LR01] B. Leimkuhler \& S. Reich, A reversible averaging integrator for multiple time-scale dynamics, J. Comput. Phys. 171 (2001) 95-114.

[LR04] B. Leimkuhler \& S. Reich, Simulating Hamiltonian Dynamics, Cambridge Monographs on Applied and Computational Mathematics 14, Cambridge University Press, Cambridge, 2004.

[Lor06] K. Lorenz, Adiabatische Integratoren für hochoszillatorische mechanische Systeme, Doctoral thesis, Univ. Tübingen (2006).

[LJL05] K. Lorenz, T. Jahnke \& C. Lubich, Adiabatic integrators for highly oscillatory second order linear differential equations with time-varying eigendecomposition, BIT 45 (2005) 91-115.

[Net00] P. Nettesheim, Mixed quantum-classical dynamics: a unified approach to mathematical modeling and numerical simulation, Thesis FU Berlin (2000).

[NR99] P. Nettesheim \& S. Reich, Symplectic multiple-time-stepping integrators for quantum-classical molecular dynamics, in P. Deuflhard et al. (eds.), Computational Molecular Dynamics: Challenges, Methods, Ideas, Springer, Berlin (1999) 412-420.

[NS99] P. Nettesheim \& C. Schütte, Numerical integrators for quantum-classical molecular dynamics, in P. Deuflhard et al. (eds.), Computational Molecular Dynamics: Challenges, Methods, Ideas, Springer, Berlin (1999) 412-420.

[PJY97] L. R. Petzold, L. O. Jay \& J. Yen, Numerical solution of highly oscillatory ordinary differential equations, Acta Numerica 7 (1997) 437-483.

[Rei99] S. Reich, Multiple time scales in classical and quantum-classical molecular dynamics, J. Comput. Phys. 151 (1999) 49-73.

[RU57] H. Rubin \& P. Ungar, Motion under a strong constraining force, Comm. Pure Appl. Math. 10 (1957) 65-87.

[Tak80] F. Takens, Motion under the influence of a strong constraining force, Global theory of dynamical systems, Proc. Int. Conf., Evanston/Ill. 1979, Springer LNM 819 (1980) 425-445.

[TBM92] M. Tuckerman, B.J. Berne \& G.J. Martyna, Reversible multiple time scale molecular dynamics, J. Chem. Phys. 97 (1992) 1990-2001.

[Zen32] C. Zener, Non-adiabatic crossing of energy levels, Proc. Royal Soc. London, Ser. A 137 (1932) 696-702. 